Horizons philosophiques

\title{
Croyances animistes et développement en Afrique subsaharienne
}

\section{Zanga Youssouf Sanogo et Nabé-Vincent Coulibaly}

Volume 13, numéro 2, printemps 2003

Herder (1744-1803) : le clair-obscur

URI : https://id.erudit.org/iderudit/801242ar

DOI : https://doi.org/10.7202/801242ar

Aller au sommaire du numéro

Éditeur(s)

Collège Édouard-Montpetit

ISSN

1181-9227 (imprimé)

1920-2954 (numérique)

Découvrir la revue

Citer cet article

Sanogo, Z. Y. \& Coulibaly, N.-V. (2003). Croyances animistes et développement en Afrique subsaharienne. Horizons philosophiques, 13(2), 139-152.

https://doi.org/10.7202/801242ar d'utilisation que vous pouvez consulter en ligne.

https://apropos.erudit.org/fr/usagers/politique-dutilisation/ 


\section{CROYANCES ANIMISTES ET DÉVELOPPEMENT EN AFRIQUE SUBSAHARIENNE}

Les débats sur les religions et la modernité dans le Tiers Monde et, en particulier, en Afrique laissent le plus souvent transparaître une certaine opposition entre les deux entités. Ainsi, d'une part, la religion, sous la forme qu'elle prend dans les sociétés dites en développement, est considérée comme un facteur rétrograde à la modernité; un frein au développement, aux libertés individuelles et à la démocratie. Curieusement, dans le cas des pays développés, la question ne semble pas poser problème, tant le développement comme fruit du progrès de la technoscience paraît avoir eu raison d'un monde autrefois dominé par la religion et des pratiques et représentations qui y avaient cours. D'autre part, la modernité symbolise le progrès, la libre expression, la démocratie et le confort technologique. Pour supporter cette thèse, très souvent on aime rappeler l'histoire de certaines entraves posées par des religieux à l'émergence de nouvelles idées qui devaient, par la suite, contribuer au progrès scientifique et technologique et à l'élargissement des espaces de liberté. On évoque, également des faits relatifs, entre autres, à la liberté individuelle, à l'émancipation de la femme, aux découvertes et au progrès scientifique et technologique.

Dans cet article, nous n'allons pas alimenter cette opposition théorique entre religions et modernité. Nous évoquons les croyances religieuses en relation avec le domaine du développement en tant que processus de changements majeurs et globaux en Afrique au sud du Sahara. Dans ce domaine, aborder le sujet de religion et de modernité reviendrait d'ailleurs à traiter du thème d'animisme et de développement, tant les attitudes et comportements des populations africaines sont, consciemment ou pas, chargés de croyances animistes, et tant la modernité rime avec la réalisation des actions de développement, la construction des infrastructures, l'adhésion à des modes de pensée émanant de la logique occidentale.

En fait, même dans les régions dites fortement islamisées ou christianisées, les réflexes et les modes de pensée animistes sont encore présents. II n'y est pas rare de voir une amulette ou des gris-gris attachés au cou, à la taille ou au bras des enfants issus de 
familles réputées pour leur foi islamique ou chrétienne. Cette réalité est d'une grande influence sur les résultats des actions de développement, de transformation et, partant, elle pose toute la question de ce que pourraient être un développement, une démocratie, une modernité africaines érigés sur des valeurs qui, malgré tout, survivent et prolifèrent au cœur des villes phares de la modernité à l'occidentale et la mondialisation aidant, s'exportent vers les métropoles du Nord. Elle impose une prise en compte des différentes logiques et visions du monde autour des actions de développement, des processus dits de démocratisation, et surtout du sort et de l'avenir d'un continent vu de l'extérieur comme en train de s'isoler et se débattre dans des problèmes d'un autre âge.

C'est dans cette perspective que nous abordons ici l'animisme. II s'agit, non pas de l'opposer à la modernité, mais d'y rechercher des éléments susceptibles d'enrichir les actions de développement des communautés africaines et de construire une modernité africaine authentique, capable de libérer toutes les potentialités de ce continent qui, malgré tout, survit et vit en dépit de toutes les analyses et tous les pronostics les plus pessimistes. Pour ce faire, il nous paraît important de comprendre d'abord la logique derrière les réflexes et modes de pensée animistes des populations rurales, urbaines, des élites politiques, administratives et religieuses.

\section{Logiques prédominantes autour}

\section{des actions de développement}

Parlant des actions de développement rural en Afrique subsaharienne, Sardan et Paquot ${ }^{1}$ faisaient la constatation suivante : "Autour des actions de développement, deux mondes entrent en contact. On peut parler de deux cultures, deux univers de significations, deux systèmes d'interprétations, comme on voudra... Disons deux systèmes de sens."

Cette diversité des systèmes de sens, selon les auteurs, serait à la base de plusieurs malentendus, voire des contradictions, autour des activités de développement. D'autres auteurs, comme Assogba et Dupré2, abondent dans le même sens. Ces contradictions et malentendus, qui étaient déjà présents dans les descriptions et analyses de l'Afrique tant par les Européens que par les Africains eux-mêmes (Achebe, Kane, Balandier, Dumont ${ }^{3}$ ), confèrent aux réalités africaines une certaine complexité qui les rend difficiles à aborder efficacement à travers les méthodes traditionnelles d'analyse et de résolution de 
problèmes, découlant de la logique cartésienne. L. S. Senghor, dans sa tentative de donner réponse à la question, la résumera de façon poétique par sa formule : «la raison est hellène, l'émotion est nègre». Nous aborderons plus loin ces méthodes traditionnelles de logique cartésienne. Nous signalons cependant que deux logiques prédominantes se dégagent autour des actions de développement et de modernisation en Afrique subsaharienne: la logique animiste et la logique moderniste.

\section{Logique animiste}

La logique animiste est celle qui prédomine notamment chez les populations rurales en Afrique subsaharienne. Elle est donc celle que partagent, le plus souvent, non seulement les agriculteurs, les éleveurs, les pêcheurs, les forgerons, les cordonniers, etc., mais aussi les responsables villageois qui dirigent les organisations paysannes et interagissent directement avec les agents de développement. Elle est également présente, à un degré plus ou moins élevé, chez les populations urbaines. Dans les villages, elle détermine, dans une large mesure, les attitudes et comportements des populations au cours de la conception et la réalisation des projets de développement économique et social. Or cette logique animiste n'est pas reconnue comme constitutive d'une représentation porteuse d'une efficacité quelconque, mais pis, elle est combattue comme l'obstacle majeur à tout progrès en Afrique. Elle est vue à travers le prisme des religions révélées comme paganisme, magie, superstition et, par les modernisateurs rationnels, comme ignorance, obscurantisme, opium du peuple. Ce schéma de lecture et d'analyse des réalités africaines a prévalu jusqu'au milieu des années quatre vingt, puis l'exemple du Japon et des dragons asiatiques a fini par égratigner, sans vraiment l'ébranler, la coquille nacrée du modèle sacro-saint 4 .

Dans la logique animiste, l'humain et son bien-être social doivent être au centre de toutes les décisions et actions posées dans la communauté. L'on considère que le bien-être social d'un individu est indissociable de celui des membres de sa famille et de celui de la communauté entière. Par ailleurs, l'on pense que la communauté des humains est celle du monde des vivants, du monde visible. Celui-ci cohabiterait avec un autre : le monde des invisibles qui comprend les puissances (fétiches), les esprits (y compris ceux des ancêtres) et les djinns. Les invisibles seraient très puissants, se manifesteraient sous des formes diverses et se trouveraient partout parmi les vivants, dans 
la forêt, dans les cours d'eau, sur les montagnes, etc. Ils auraient la capacité non seulement de veiller au bien-être des vivants, mais aussi de leur poser des problèmes quand ils sont fâchés. D'où l'existence des rites, des sacrifices propitiatoires, des tabous et des règles de conduite sociale, afin que les vivants continuent de bénéficier de la grâce et de l'assistance des invisibles dont le rôle premier et la fonction sont la médiation.

Dans cet animisme, les puissants sont en fin de compte des intermédiaires entre les vivants et Dieu qui, sans être désigné par le même nom que celui des religions monothéistes révélées, est le plus souvent le destinataire final des incantations et prières. Ainsi, les Dogons réfèrent à $\mathrm{Amma}$, les Bamanan à $\mathrm{Ngala}$ et le Sénoufo ${ }^{5}$ à Klè et, dans tous les cas, il s'agit d'un dieu unique créateur et garant de l'ordre du monde. Un monde inachevé, confié à l'homme qui doit apprendre à en user sans abuser, car lui-même est créature parmi d'autres créatures, force parmi d'autres forces, ni meilleur, ni pire, ni supérieur, ni inférieur, seulement différent, ce qui n'enlève rien à son génie. Un tel homme n'est jamais un individu, mais une personne, un faisceau de liens multiples et multiformes, un nœud de réseaux, un carrefour de forces qui doit apprendre à survivre et vivre dans un monde en perpétuel mouvement et recherche d'équilibre. Dans un tel contexte, "devenir maître et possesseur du monde" frise la folie furieuse, le déni de réalité ou une naïveté juvénile. Pour l'animisme, l'homme peut tirer beaucoup de profits du monde; il peut réussir à parler aux choses, aux êtres, à Dieu, et se forger une personnalité formidable (belle et terrifiante), mais en bout de ligne l'ordre du monde, sa loi fondamentale aura raison de lui. Vivre c'est évoluer dans un monde qui peut toujours nous surprendre, voire nous terrasser. La durée d'une vie ne suffit pas pour apprendre du monde et apprendre le monde; il nous faut le concours des autres sans lesquels chacun est terriblement limité. "En naissant j'étais dans leurs mains, en mourant je serai dans leurs mains", disent les Bamanan du Mali.

Cette croyance animiste, qui découle des us et coutumes, est à la base du système d'enseignement traditionnel. II faut dire que les traditions africaines, les croyances et philosophies éducatives qui en découlent, rejoignent, à bien des égards, celles observées ailleurs dans le monde: en Inde, au Japon ou en Chine ${ }^{6}$. Parlant de l'Afrique subsaharienne, des auteurs comme Moumouni, Coulibaly, Ajayi, Mazrui et Wagaw, Diawara et Djangrang 7 révèlent que les populations accordent une grande importance à l'humain et au social. On y 
recherche constamment une certaine harmonie entre la société et la nature, entre le monde des vivants et celui des morts, entre le visible et l'invisible. L'on considère que l'homme, la nature et Dieu s'interpénètrent. Donc vivre c'est comprendre (au sens latin comprehendere) autant que possible la nature, le monde, le divin, pour éviter de commettre des erreurs fatales. D'où le respect, les précautions de langage, de comportement et d'attitude de l'animiste que certains auteurs considèrent à tort comme des peurs superstitieuses, un besoin de fusion avec le monde, une pensée magique.

Dans un récent article, Sanogo ${ }^{8}$ aborde largement les finalités éducatives poursuivies à travers le système d'enseignement traditionnel, issu des croyances locales africaines, forgées et imprégnées par l'animisme. II ressort qu'il se distingue surtout par sa tendance sociocentrique. Sa finalité est alors, non pas de donner nécessairement une indépendance maximale et autarcique à l'individu, mais de le préparer essentiellement à servir la société, à y favoriser une vie plus harmonieuse, à remplir un rôle honorable et constructif dans la communauté. II vise à construire une personne forte et souple, capable de se démarquer comme individu, et de vivre en complémentarité avec les autres, avec la nature, car dans l'animisme, c'est en tant qu'individu qu'on laisse son nom dans l'histoire et mérite le statut d'ancêtre. L'humain peut apporter son génie propre à la société, mais pour que celui-ci soit utile, reconnu et proposé à la postérité, il doit faire avancer la communauté humaine. L'homme idéal de l'animiste est un héros au sens grec, doublé d'un philosophe, (Socrate et Ulysse en un). II a une personnalité éprouvée et dotée de la capacité du négociateur et du rassembleur. C'est un chef capable de conduire une communauté avec droiture et justice, valeurs dont on mesure peu l'importance pour les populations dans le monde politique africain.

Outre ce sociocentrisme, le système d'enseignement traditionnel présente deux autres tendances : théocentrique et écocentrique. II est théocentrique car il accorde une certaine importance au divin et au respect des règles de conduite dictées par les croyances animistes locales. Dans le panthéon animiste, il y a Dieu, source et finalité du monde, mais un dieu distant, qui n'est pas appelé à intervenir dans le cours des événements quotidiens car l'être humain dispose d'une intelligence pour gérer sa vie. D'où le besoin d'intermédiaires entre lui et les humains, par commodité de communication et par respect. Nous retrouvons là toutes les dimensions de ce que G. Van Der 
Leeuw ${ }^{9}$ nomme l'objet de la religion sauf le livre sacré. Nous pouvons donc conclure que l'animisme a les traits et caractères essentiels de toute religion, mais surtout qu'il commande chez ceux qui le pratiquent des comportements et une attitude morale qui vont au-delà des conventions sociales, du respect des choses, des êtres, de soi et des autres. Quant au caractère écocentrique, il fait référence au souci de rechercher une certaine compréhension globale écologique : connaître la nature et l'univers, indépendamment du service rendu à la société ou aux divinités. Mazrui et Wagaw ${ }^{10}$ expliquent que l'écocentrisme traditionnel africain est dominé par un certain souci pour l'environnement. L'Africain essaierait de comprendre l'écologie pour mieux vivre en harmonie avec son environnement. Toutefois, il est difficile de départir ces deux tendances, tant l'écocentrisme traditionnel repose sur des considérations divines ou religieuses.

La prédominance des enseignements traditionnels sociocentriques, théocentriques et écocentriques, qui caractérisent la personnalité africaine, semble avoir favorisé le développement d'une sorte de pensée qu'on pourrait qualifier de globale ou holistique. Cela se révèle, par exemple chez les populations rurales, à travers leurs analyses des situations de développement et leurs prises de décisions pendant les réunions au village. Au cours de ces réunions, on se rend compte que les décideurs villageois font généralement le lien entre les projets de développement soumis et beaucoup d'autres facteurs propres au village. Ils anticipent l'impact des projets proposés sur la société. En fait, dans leurs analyses et prises de décisions, de façon implicite ou explicite, ils se doivent, entre autres, de considérer l'humain et le social, de prévenir les réactions des ancêtres et des autres invisibles, et de se soucier de leur environnement, de l'avenir de ceux qui viendront après nous, bref du développement durable des théories modernes et post-modernes. Certes, au cours des réunions publiques avec les agents de développement, ils acceptent parfois de dévoiler certains aspects des principes et règles de conduite du village, mais nombreux sont ceux qui sont gardés en secret. Dévoilés ou pas, de leur respect dépendent l'adhésion des populations aux projets et la réussite des activités.

Par ailleurs, il convient de noter que les communautés africaines rurales ne vivent pas en vase clos et ne sont pas stationnaires : comme toute communauté, elles évoluent. À titre d'exemple, on peut citer les peuples qui sont passés des outils rudimentaires traditionnels au tracteur motorisé et à la sélection des semences. Même si certaines pratiques animistes ont encore cours, dans nombre de 
villages, beaucoup de rites, naguère régulièrement célébrés, ont été abandonnés avec le temps. Cependant, la cessation d'un rite n'est pas synonyme de l'absence des croyances qui y sont liées. Souvent, même longtemps après la cessation d'une pratique, les tabous continuent à être respectés, les règles de conduite aussi.

\section{Logique moderniste}

Lorsque nous parlons de logique moderniste, nous désignons celle qui est généralement partagée par les agents de développement. II s'agit notamment des agents de terrain: entre autres, les techniciens d'agriculture, de la santé, d'élevage et de pêche. II s'agit également des technocrates qui, d'une manière ou d'une autre, décident de la politique agricole ou du développement socioéconomique des villages, des villes, des pays: administrateurs, bailleurs de fonds, ingénieurs, politiciens, experts, consultants. Qu'ils soient du terrain ou du bureau, africains ou pas, les paysans, qui ne manquent pas d'humour, aiment souvent dire d'eux qu'ils ont appris à transformer cinq francs en dix francs, à partir des haillons pour arriver aux costumes, à faire du chaud en partant du froid et vice versa. Sortis des écoles de formation, ces agents ont reçu une éducation à prédominance occidentale. Ils ont appris à analyser et à résoudre le plus souvent en théorie - les situations et les phénomènes en faisant constamment recours aux recettes scientifiques et aux techniques modernes. Leur vision du monde est dominée par la logique scientifique cartésienne, supposée plus objective et plus efficace en matière de changement et de transformation du monde et de la société. Pour eux, la religion, les croyances animistes sont des obstacles idéologiques car les populations leur donnent trop d'importance, s'en servent trop pour juger et évaluer la valeur des changements proposés et souhaités dans leur vie.

Dans la logique cartésienne, symbole et modèle de la modernité, n'est valide que ce qui est objectif, observable, vérifiable ou démontrable. L'on pense que, pour être valable, une méthode, une représentation doit être scientifique, permettre d'obtenir des résultats objectifs et aboutir à des recettes généralisables. On y accorde une plus grande importance au concret, au rationnel, au mesurable. L'invisible, le mystique, l'indémontrable est considéré comme irrationnel, subjectif, donc non fiable.

Sanogo ${ }^{11}$ présente les finalités éducatives du système d'enseignement de type occidental. Celui-ci (selon Mazrui et Wagaw ${ }^{12}$, cités par l'auteur) se caractérise surtout par ses tendances 
égocentrique et écocentrique. Son égocentrisme se manifeste par la grande importance accordée à l'accomplissement de l'individu et à son propre épanouissement. Quant à son écocentrisme, il se présente, non pas comme un souci pour l'écologie, mais comme une curiosité pour comprendre cette dernière, la maîtriser, voire la transformer et la contrôler de façon à favoriser l'accomplissement de soi, l'épanouissement socio-économique. Cette philosophie qui caractérise le système d'enseignement de type occidental le distingue de la formation traditionnelle africaine. Et toute la philosophie éducative qui le sous-tend semble rimer avec la vision égocentrique et la curiosité écologique. Contrairement à l'éducation traditionnelle africaine, le plus souvent tout ce qui échappe à la logique scientifique, tel que le monde des invisibles, est banalisé.

Nyéréré, Mazrui et Wagaw et Ki-Zerbo'13 reprochent à l'enseignement de type occidental d'avoir éloigné les écoliers des normes et valeurs africaines, de cultiver le mépris des traditions et de favoriser l'élitisme et l'individualisme dans une société où la vie communautaire est une tradition. Par ailleurs, au-delà de ces reproches, Le Moigne, Checkland, Senge et Gautier ${ }^{14}$ remettent en cause la tendance à appliquer les méthodes traditionnelles d'analyse et de résolution de problèmes aux situations problématiques, dont la complexité se prête moins à leur utilisation. Ils relèvent leurs limites quant à ces situations, notamment leur caractère linéaire, privilégiant des relations de cause à effet et réductionniste, limitant l'analyse des situations à quelques variables. Ils recommandent plutôt le recours à des méthodes souples et systémiques qui prennent en compte le caractère relatif des situations problématiques à l'étude. Nous signalons que, loin de remettre en cause le mérite des méthodes scientifiques cartésiennes dans le progrès accompli par l'humanité, nous sommes d'accord avec les auteurs pour relever leur caractère inapproprié pour des situations problématiques complexes, comme celle des interventions pour le développement, où les subjectivités des acteurs sont pratiquement incontournables, si l'on veut aboutir à des résultats viables et acceptables par tous.

On comprend donc mieux pourquoi l'animisme, qui refuse de dépouiller le monde de son âme et de son sens, qui s'évertue à vouloir un monde où tout se tient dans une dialectique de différentiation et de complémentarité, est accusé de syncrétisme douteux, de bricolage irrationnel et contre productif. Le non développement de tout un continent est mis à son actif (Kabou, Kä Mana, Manguele ${ }^{15}$ ). Mais 
cela pose une question, à notre avis, cruciale. Si l'Afrique a du mal à danser avec les habits et les masques d'un autre, pourquoi n'en fait-elle pas d'autres à sa mesure? Car tout est là, bon ou pas; la personnalité africaine est façonnée et imprégnée par l'animisme et aucun volontarisme, fut-il prométhéen, ne semble pouvoir y changer grand chose. Mieux, ceux qui se font les champions de la modernité sont vite rattrapés par leur substrat animiste à la moindre tempête qui souffle sur leur vie, faisant sourire à l'envie les vieux du haut de leur sagesse traditionnelle qui prêche que "se connaître vaut mieux que savoir monter à cheval, et savoir monter à cheval vaut mieux que connaître le monde". D'où la question suivante: en quoi l'animisme comme fond culturel, philosophique et religieux africain peut-il contribuer à forger les stratégies et les outils d'un développement à l'africaine? De même que le christianisme a façonné l'homme et la civilisation occidentale, de même l'animisme africain coexiste au cœur de l'islam et du christianisme en Afrique, bien que combattu par l'un et l'autre, avec ou sans l'appui de l'idéologie du développement des modernistes locaux et étrangers.

\section{Animisme, religion et changements en Afrique}

Que l'animisme soit une religion aussi bien dans son objet que dans sa fonction qui est de donner un sens à la vie humaine, l'expérience de l'Asie et de l'Afrique analysée par les études sur la question le démontre bien (Van Der Leeuw, Kä Mana, Helena Norberg-Hodge ${ }^{16}$ ). Loin d'être de pures attitudes de résistance ou de refus du changement et du développement, les comportements inspirés par l'animisme traduisent une philosophie de la vie, de l'être humain, un projet de société, un mode de développement.

En enseignant des valeurs de respect de la nature et de tout ce qu'elle contient, l'animisme se trouve prêcher pour l'écologie qui est devenue une évidence pour tous les pays développés ou non. René Dumont et Hugues Dupriez ${ }^{17}$ montrent bien qu'avec les types de sols qu'ils ont, les paysans africains utilisent les outils les plus adaptés et les techniques culturales les plus performantes. Au Kenya, les colons fermiers anglais l'ont appris à leurs dépens en introduisant la charrue à labour profond, qui a failli stériliser les sols en ramenant à la surface la latérite. Malgré leurs limites, les paysans connaissent bien leurs sols et la façon de les exploiter sans les ruiner. Ils cherchent à transformer sans abîmer, à changer dans la continuité, comme d'autres veulent éduquer sans abîmer (Ki-Zerbo ${ }^{18}$ ) ou moderniser sans exclure (Bertrand Schwartz ${ }^{19}$ ). Selon Boubou Hama ${ }^{20}$, "ce respect des 
choses et des êtres donne à l'univers sa stabilité et à l'être son équilibre lié à celui de l'univers qui le porte", et cela permet à l'homme de réaliser son "tout" et d'être en accord avec la nature et avec lui-même.

Sur le plan social, politique, spirituel, nous retrouvons les mêmes soucis et principes qui veulent que chacun soit respecté pour ce qu'il est, sans jamais oublier qu'il appartient aussi aux autres qui peuvent exiger de lui sans s'emparer de sa personne. C'est dans cette perspective que le politique est compris et abordé comme une palabre, où chacun doit être reconnu, représenté avec droit de parole et responsabilité d'engagement une fois la décision prise. II n'est donc pas étonnant que, pour la majorité des populations africaines, la politique dominée par les élites modernisantes soit une appropriation inacceptable et une insulte à leur être et leur personne. Le premier défaut de la politique en Afrique se révèle alors le déficit de dialogue et de justice sociale qui reposent avant tout sur la reconnaissance et le respect des personnes et de leurs droits. Là encore, on ne peut s'empêcher de penser à ce besoin de démocratie directe et participative qui est de plus en plus réclamée dans les pays développés.

II est certain que l'animisme n'offre pas la solution à tous les problèmes qui assaillent l'Afrique aujourd'hui, car il a ses propres limites, comme la trop grande prudence qui frôle parfois le conservatisme. Cependant, sa vision intégrale de l'être humain, son souci de préserver les équilibres sociaux, naturels, voire cosmiques, incitent à une forme de sagesse nécessaire à notre monde, qui entre dans une phase de questionnement et de remise en cause de ses pratiques modernes. Nous pensons qu'à ce titre il constitue une base commune aux cultures africaines, à partir de laquelle des politiques et stratégies de changement et de développement ont plus de chance de prendre racines, d'être significatives sur le plan pratique et symbolique pour une majorité appelée à y contribuer. L'animisme offre, en effet, une ouverture et une souplesse pour inventer et appliquer des méthodes de gestion de la diversité sociale, naturelle, politique et religieuse, qui sera à coup sûr un des défis majeurs du nouveau siècle et face à laquelle les systèmes traditionnels de la modernité montrent des insuffisances, des lacunes.

Le gigantisme monstrueux des modèles modernistes et leur mode de fonctionnement lourd, complexifié et quasi occulte (mystérieux et rébarbatif), pour les non-initiés, génèrent des attitudes et comportements d'auto-exclusion, de révolte, de non-confiance et de rejet de la 
part des citoyens ordinaires. Les outils et mécanismes rationnellement standardisés et harmonisés par la modernité pour plus d'efficacité ont certes résolu bien des problèmes et comblé autant de besoins, mais c'est comme si ce succès a fait perdre de vue que la réalité se laisse difficilement enfermer dans des cadres ou, comme disaient les philosophes anciens, que l'être est ondoyant et divers. La complexité des problèmes post-modernes montre qu'on ne peut gérer la diversité avec les mêmes outils habituellement utilisés pour agir sur l'homogénéité. D'où le proverbe africain selon lequel «diriger des hommes ne consiste pas à les battre tous avec le même bâton». En d'autres termes, il convient de modifier les approches et méthodes de gestion en fonction de la spécificité des situations. Dans le cas de l'Afrique, quarante ans de résultats mitigés de coopération et de développement exigent de penser et de faire les choses autrement.

La mise en œuvre des projets de développement et leurs évaluations restent encore largement empruntes de la logique scientifique cartésienne des élites modernisantes locales et internationales. Peu d'importance est accordée à la logique animiste paysanne. Cette réalité a suscité, et suscite encore, des reproches chez différents auteurs qui se sont penchés sur la problématique des interventions pour le développement. Ces derniers privilégient notamment le recours aux sciences sociales non économiques (sociologie, anthropologie, ethnologie, etc.) pour fléchir la rigidité scientifique et technocratique, dont l'application sans discernement dans les quatre décennies de l'aide au développement de l'Afrique a donné l'impression qu'on a navigué à vue, expérimenté, tâtonné et erré à hue et à dia.

Si opposer religion et modernité peut servir à alimenter les discussions et échanges entre amis, des conférences sur les campus universitaires, il convient de reconnaître qu'il pourrait plutôt compromettre la réussite des projets dans le domaine du développement et des changements. Dans le cas des pays africains au sud du Sahara, dont les populations sont marquées et pétries par les enseignements tirés des traditions animistes, il est préférable d'exploiter les logiques et recettes locales, dans une perspective d'enrichissement des décisions et actions de développement et de transformation des sociétés. II faudrait également être conscient que l'Afrique ne refuse pas le changement et la modernisation; son histoire récente en témoigne du reste, mais elle regarde, discute les modalités et la finalité de tout changement. Le changement proposé 
doit respecter une certaine continuité et non vouloir refonder ou recommencer le monde. La précarité des équilibres de toute nature sur le continent a sans doute à voir avec cette extrême prudence.

Par ailleurs, compte tenu de leur fragilité, on ne saurait rejeter les équilibres acquis sans avoir au préalable la garantie que les nouvelles solutions ou manières de faire proposées sont effectivement meilleures et plus durables. Les enjeux sont trop cruciaux, trop vitaux, pour qu'on s'amuse à jouer le sort de populations entières et l'avenir de communautés vieilles de plusieurs centaines ou milliers d'années à la roulette russe. Le plus important ne doit point être l'origine des savoirs et pratiques proposés, mais leur contenu, leur viabilité par rapport aux situations, aux problèmes et leur acceptabilité par l'ensemble des acteurs. Donc, peu importe qu'ils émanent d'une proposition paysanne ou d'une théorie scientifique. Le défi des agents de développement réside alors dans l'intégration réelle de la logique locale dans leurs pratiques, et dans la recherche du viable pour l'ensemble des acteurs, au moins pour la majorité. Cela suppose qu'on tienne compte des forces et réalités locales réelles, matérielles, spirituelles, symboliques, sociales, pour ne pas réduire l'être humain à un ventre, ou pis, à un zombie en le vidant de son être subtil, de sa substance. Si l'Asie de développe en respect avec ses valeurs culturelles et religieuses, que l'Occident est à la recherche d'un supplément d'âme parce que forcé de donner raison à Rabelais (science sans conscience n'est que ruine de l'âme), comment imaginer que l'Afrique doive jeter ou vendre la sienne pour embrasser, acheter la modernité? De plus, en quoi les cultures africaines basées sur des archétypes animistes sont-elles si éloignées des nouvelles visions et modèles de développement que les problèmes les plus graves de la modernité font naître de plus en plus dans les pays développés? Et si l'Afrique était le continent de l'avenir parce qu'il "constitue une immense réserve de sens et de ressources 21 "?

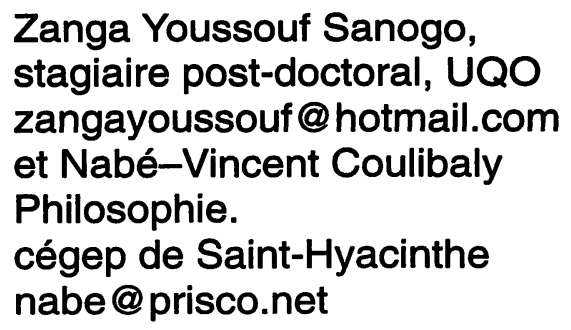


1. J. P. O. D. Sardan et E. Paquot, D'un savoir à l'autre : les agents de développement comme médiateurs, Paris, GRET, 1991, p. 17.

2. Y. Assogba, «Le paradigme interactionniste et le processus du développement communautaire : l'exemple des ONG en Afrique", in Revue canadienne d'études et du développement, vol. IX, no 2, p. 201-218; G. Dupré, Savoirs paysans et développement, Paris, Karthala-ORSTOM, 1991.

3. C.A. Kane, L'aventure Ambiguë, Paris, Julliard, 1994. C. Achebe, Le monde s'effondre, Présence africaine, 1964; R. Dumont, L'Afrique Noire est mal partie, Paris, Seuil, 1962, 1966; G. Balandier, L'Afrique ambiguë, Paris, Terre humaine/Plon, 1969.

4. B. Bolya, L'Afrique à la Japonaise, Ivry-sur-Seine, Editions Nouvelles du Sud, 1994.

5. Dogons et Bamanan sont des ethnies du Mali, mais on retrouve les Sénoufo aussi bien au Mali qu'au Burkina Faso et en Côte d'Ivoire.

6. Lire respectivement les ouvrages suivants : A. S. Gnanasambandan, «Inde : les cycles de la réincarnation", in Le Courrier de l'Unesco, mars 1998, p. 14-18; J. Clammer, "La politique animiste", in Interculture, cahier no 137, avril 2000, p. 21 45; F. Aubin, "Chine : la gestion du surnaturel», in Le Courrier de l'Unesco, mars 1998, p. 10-14.

7. A. Moumouni, L'éducation en Afrique, Paris, François Maspero, 1967; N.V. Coulibaly, "Ce que l'Afrique d'hier peut dire au monde d'aujourd'hui", in Interculture, cahier 78, vol. XVI, no 1, janvier-mars 1983, p. 2-15; J.F.A. Ajayi, "L'éducation dans l'Afrique contemporaine : historique et perspective", in Le processus d'éducation et l'historiographie en Afrique, (documents de travail et compte rendu du colloque organisé par l'Unesco à Dakar, Sénégal, du 25 au 29 janvier 1982), p. 11-22, Paris, Unesco, 1986; A.A. Mazrui et T. Wagaw, "Vers une décolonisation de la modernité : éducation et conflits culturels en Afrique de l'Est", in Le processus d'éducation et l'historiographie en Afrique, (documents de travail et compte rendu du colloque organisé par l'Unesco à Dakar, Sénégal, du 25 au 29 janvier 1982), p. 35-64, Paris, Unesco; 1986; I. Diawara, "Cultures nigériennes et éducation : domaine Zarma-Songhay et Hausa", in Présence africaine, nouvelle série bilingue, no 148, 1988, p. 9-19; N.B. Djangrang, "Afrique : les souffles de l'univers", in Le Courrier de l'Unesco, mars 1998, p. 6-9.

8. Y. Sanogo, "Valeurs et interventions éducatives: cas du développement rural en Afrique subsaharienne", in Horizons philosophiques, printemps, vol. 12, n० 2, 2002, p. 101-114.

9. G. Van Der Leeuw, La religion dans son essence et ses manifestations, Paris, Payot, 1970.

10. Auteurs cités plus haut.

11. Loc. cit.

12. Auteurs cités plus haut.

13. J. Nyéréré, Indépendance et éducation, Yaoundé, Cameroun, Éditions CLE, 1972; J. Ki-Zerbo, (sous la direction de) Éduquer ou périr, Paris, Unicef-Unesco, 1990; Mazrui et Wagaw, déjà cités.

14. J.L. Le Moigne, La théorie du système général : théorie de la modélisation, Paris, Première édition, Presses Universitaires de France, 1977; P. Checkland, Systems Thinking, Systems Practice, Toronto, John Wiley \& Sons Ltd, 1981; P. Senge et A. Gautier La cinquième discipline, (traduit et adapté de l'américain par Hervé Plagnol), Paris, First, 1991. 
15. A. Kabou, Et si l'Afrique refusait le développement? Paris, L'harmattan, 1991; K. Mana, L'Afrique va-t-elle mourir?, Paris, Cerf, 1991; D.E. Manguele, L'afrique a-telle besoin d'un ajustement culturel?, Ivry-sur-Seine, Editions Nouvelles du Sud, 1990.

16. N. Norberg-Hodge, Quand le développement crée la pauvreté, Paris, Fayard, 2002; Van Der Leeuw et Kä Mana cités plus haut.

17. René Dumont, op. cit.; H. Dupriez, Paysans d'Afrique, Paris, L'harmattan et Editions Terres et vie, 1988.

18. Op. cit.

19. Bertrand Schwartz, Moderniser sans exclure, Paris, Découverte/essais, 1994.

20. Boubou Hama, cité par Hugues Dupriez (cité ci-dessus).

21. J.M. Ela, Innovations sociales et renaissance de l'Afrique noire, les défis du monde d'en bas, Paris/Montréal, L'harmattan, 1998. 